\title{
Evaluation Model of Power Retail Market Construction Effectiveness
}

\author{
Zhang Han $^{1, *}$, Xue Song ${ }^{1}$, Hu Yuan ${ }^{1}$, Ma li $^{1}$, Zhang Xiaoxuan ${ }^{1}$ \\ ${ }^{1}$ Dept. of Corporate Strategy State Grid Energy Research Institute Company Limited
}

\begin{abstract}
With the deepening of the power system reform since 2015, the construction of the electricity retail market continues to accelerate, the activity and competition of the electricity retail market continues to increase. In order to better guide the construction and development of China's electricity retail market, it is necessary to accurately evaluate its construction effectiveness, finding the effectiveness and deficiencies, and making electricity retail market play the decisive role in the allocation of electricity resources. This article proposes a new set of construction effectiveness evaluation model for electricity retail market.It establishes the key factors of power retail market construction effectiveness from the seven aspects of market mechanism, market openness, market concentration, market quality, market performance, electricity retail side service content diversity and market satisfaction, etc. The corresponding evaluation index system is formed to measure the effectiveness of the electricity retail market construction, which helps to guide the discovery of weak links in retail market construction of power system and improve them in a timely manner. The validity of the proposed model is proved by case study.
\end{abstract}

\section{Introduction}

On March 15, 2015, "Several Opinions on Further Deepening the Reform of the Electric Power System" was issued, marking the official start of a new round of power system reform in China [1]. Among them, the electricity retail side reform is one of the highlights of the new electricity reform and one of the core tasks. It aims to form an "more buyer, more seller" market structure by orderly opening up the electricity retail business and build an effective competitive electricity retail market structure and system,making the market play the fundamental role in allocating resources and discovering the true value of electricity.

In general, the current electricity retail market in China presents the following competitive situation. First, the scale and capacity of the electricity retail market is gradually increasing. The continuous liberalization of electricity generation as well as utilization plans and the increase in the scale of the electricity retail market have attracted a large number of private capital injections, bringing more risks to the secure operation of power system, which needs to be cautioned [2-3]. At present, more than half of the electricity retail companies established are independent companies invested by social capital. Second, the power disparity between electricity retail companies is huge. Power generation companies have strong market control in the current market transactions, and most of them have a relatively fast business development and strong ability to grasp the market. Third, the operating pressure of electricity retail companies has increased, and the high-spread situation in the initial stage of the electricity retail market no longer exists. The profit proportion of power users is increasing, and the profit margins of power generation companies are constantly decreasing, which has caused the profit of power retail companies to be compressed [4]. Many power retail companies are difficult to run actual power retail business or even withdraw from the market.

It is very necessary to study a scientific and reasonable evaluation method for the effectiveness of the electricity retail market construction that suits China's national conditions. However, the current research on the evaluation model and method of the electricity retail market construction is still very imperfect. The lack of relevant evaluation index system cannot effectively measure the actual operation level of the market and timely discover the weaknesses and deficiencies. To a certain extent, it has restricted the continuous improvement and development of the electricity retail market.

Based on the advanced experience of foreign electricity retail market construction effectiveness evaluation, this article analyzes the key influencing factors of the orderly development of China's electricity retail market, innovatively proposes the theoretical model and method of China's electricity retail market construction effectiveness evaluation. An example is given to verify the validity and practicability of the proposed model.

\footnotetext{
* Corresponding author: augustzh@163.com
} 


\section{Key factors for the effectiveness of the electricity retail market}

To evaluate the effectiveness of the electricity retail market construction, we must first reasonably determine the key factors for the effectiveness of the electricity retail market construction, systematically split the major problem into several small problems, and reduce the difficulty of analysis while enhancing rationality, effectiveness and operability of the evaluation. Starting from the dimensions of policy system, mechanism design, market operation, competition level, service content, and service quality, this article establishes the key factors for the effectiveness of the electricity retail market from 7 aspects, which are market mechanism,market openness, market concentration, market quality, market performance, the diversity of service content on the electricity retail side and market satisfaction. The details of the 7 key factors are as follows.

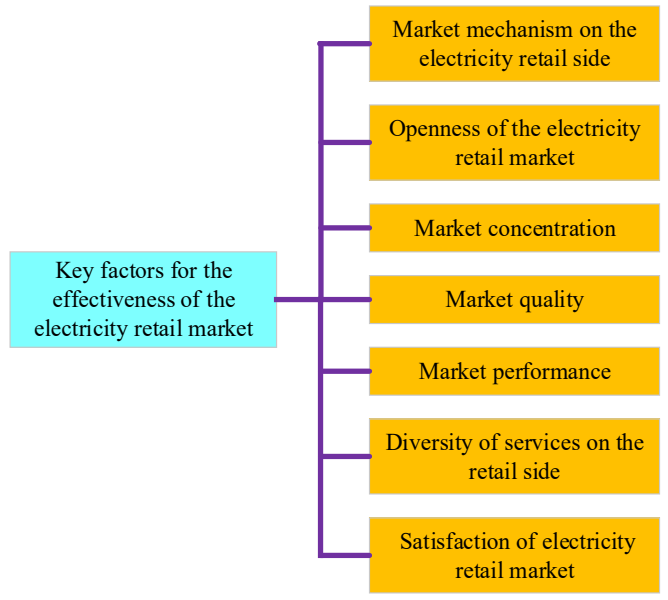

Figure 1. Key factors for the effectiveness of the electricity retail market

\subsection{Market mechanism on the electricity retail side}

China's power system reform is mainly driven by government forces from top to bottom. Therefore, before the market role is fully exerted, good market mechanism design plays a vital role in the cultivation as well as the stable and healthy development of the electricity retail market. A good market mechanism can effectively increase the enthusiasm of various market players to participate in the market, continue to promote the expansion of the market scale, strive to maintain the fair and open operation of the market, and improve market competition efficiency. Therefore, the power retail side market mechanism is an important criterion for evaluating the progress of power retail market reforms.

\subsection{Openness of the electricity retail market}

The goal of the market-oriented reform of the electricity retail side is to establish an open electricity retail market and increase the freedom along with convenience of electricity trading. The degree of liberalization of market-oriented users and the progress of market construction largely determine the activity of the electricity retail market. The continuous liberalization of the operating power generation and utilization plan, the further relaxation of market access conditions and the reduction of the electricity consumption cost help to attract more diversified market players to enter the electricity retail market. Various electricity retail companies, incremental distribution operators, integrated energy service providers and large power users, have brought the necessary competitiveness to the construction of the electricity retail market.

\subsection{Market concentration}

Relying on the advantages of natural capital, and technology, large electricity retail companies with the background of power generation groups occupy a large market share in various places, which limits the healthy and sustainable development of private and other electricity retail companies. This article uses market concentration to measure the competitiveness of the electricity retail market. Market concentration is a measure of the concentration of the market structure of the entire industry. Market concentration is the most basic and important factor that determines the market structure, and embodies the degree of competition and monopoly in the market [5].

\subsection{Market quality}

The quality evaluation of the electricity retail market can quantitatively and intuitively display the evaluation results of the electricity retail market construction, which helps decision-makers understand the characteristics of the electricity retail market in depth and provide references for them to formulate strategies to improve market quality. The quality evaluation of the electricity retail market can be carried out from the current level of the market, the development speed of the market, the stability of the market, the electricity retail efficiency and the market structure,etc.

\subsection{Market performance}

The performance of the electricity retail market is a key factor to measure whether the electricity retail market meets the expected construction goals, mainly to measure the effectiveness of the electricity retail market construction. The performance of the electricity retail market comprehensively and systematically quantifies the effectiveness of the construction of the electricity retail market in terms of resource allocation level, industrial structure distribution and scale, innovation and technological progress, and the input-output ratio of enterprises. It is the core evaluation element. 


\subsection{Diversity of services on the retail side}

At the initial stage of the liberalization of the electricity retail market, electricity retail companies can obtain good income by selling electricity. As the profit margin of the power generation side continues to decline and the share of users continues to increase, the traditional business model of electricity retail companies is difficult to maintain. It is necessary to expand the service content of the electricity retail side and enrich the service model. The core value-added electricity retail service improves the profit level and the operation strength.

\subsection{Satisfaction of electricity retail market}

As more and more market players enter the electricity retail market to compete, electricity retail companies must increase customer satisfaction if they want to maintain the stability and continuity of their business. It is important to strengthen customer stickiness, expand incremental customers, and avoid customer churn. When users' feedback and choices can guide various market players to make adjustments, an open electricity retail market with users as the core has taken shape.

\section{Evaluation model for the effectiveness of the electricity retail market}

\subsection{Index system for evaluating the effectiveness of the electricity retail market}

Based on the systematic analysis of the key factors for the effectiveness of the electricity retail market construction, various key factors are further deepened to construct an evaluation model. An evaluation index system is formed from seven aspects, and a comprehensive evaluation of the construction effectiveness can be made.

\section{A. Market mechanism}

This type of evaluation index is divided into five aspects: electricity price system, transaction mechanism, supervision mechanism, entry and exit mechanism and guaranteed power supply mechanism.

\section{B. Market openness}

This type of evaluation index is mainly evaluated by five indicators: the market-oriented user liberalization ratio, the diversification degree of electricity retail entities, the entry and exit ratio of market entities, the correlation between terminal electricity prices and spot market prices, and the exit costs of electricity retail enterprises.

\section{Market concentration}

The concentration of the electricity retail market are mainly focused on the electricity retail side and the users' side, through $\mathrm{HHI}$ index and user conversion rate.

\section{Market quality}

It is mainly evaluated through five aspects: the current level of the market, the development speed of the market, the stability of the market, the electricity retail efficiency, and the market structure.

\section{E. Market performance}

It is mainly evaluated through six aspects: industry resource allocation efficiency, industry scale structure efficiency, industrial technology progress, electricity retail enterprise input cost, electricity retail enterprise input-output ratio, and user conversion cost.

\section{F. Diversity of services on the retail side}

It is mainly evaluated through four indicators: the diversity of electricity retail packages in the market, the degree of value-added service development, the degree of demand-side response participation, and the type and number of independent service providers.

\section{G. Satisfaction of electricity retail market}

It is mainly evaluated through four indicators, such as the satisfaction degree of power users, the complaint rate of power retail companies, the frequency of users changing power retail companies, and whether the user feedback channel is smooth.

\subsection{Evaluation methods for the effectiveness of electricity retail market construction}

On the basis of the above evaluation index system, the following five steps are taken for competitiveness diagnosis and evaluation.

Competitor selection. Select the international advanced electricity retail market and domestic electricity retail market.

Data collection. The data is mainly obtained from government official websites, social evaluations, industry analysis reports, regulatory department reports, and related materials.

Weight setting. Through questionnaire surveys, expert interviews and other methods, AHP and expert weighting methods are used to determine the weights of indicators at all levels.

Score evaluation. According to the principle of combining subjective and objective, the evaluation is carried out by refining the grading level, clarifying the scoring standard, and organizing expert scoring.

Diagnostic analysis. According to the evaluation results, the effectiveness of the electricity retail market construction is evaluated, and the improvement strategy is proposed.

The data of China's target electricity retail market is selected for diagnostic analysis, and the mature electricity retail market of developed countries with high international visibility and high influence, such as Texas, United Kingdom, and Australia, is selected as the 
benchmarking object. According to the specific index data, the secondary dimension index is assigned a comprehensive value of 1 to 5 points. 5 is excellent, 4 is good, 3 is average, 2 is poor, and 1 is very poor.

Accumulate the scores of different indicators and the product of weights to obtain the final score of the effectiveness of electricity retail market.

\section{Case study}

Take the electricity retail market in Province A as an example to diagnose the effectiveness of its electricity retail market construction, through benchmarking with foreign mature electricity retail markets (Texas electricity retail market in the United States). Based on empirical research results, we analyze the weak links of electricity retail market construction, and propose an orderly improvement strategy and implementation recommendations for the electricity retail market from the perspective of government authorities. From the seven aspects mentioned above, the effectiveness of the electricity retail market construction in Province A and Texas is evaluated. The evaluation results are shown in Figure 2.

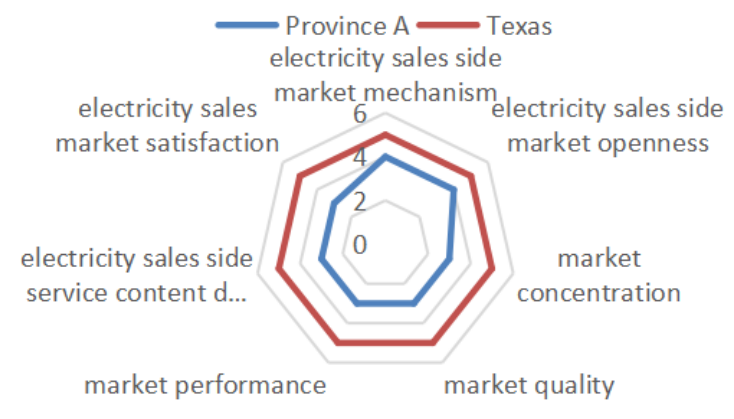

Figure 2 The evaluation results effectiveness of the electricity retail market construction in Province A and Texas

As shown in the figure, the construction of the electricity retail market in Province A is lagging behind that in Texas. It only scored 4 points on the electricity retail market mechanism and electricity retail side market openness, which are relatively close to the Texas' s level. It is recommended that Province A continues to strengthen the construction of the electricity retail market in the future and enrich relevant policy requirements and market mechanisms. Strengthen the cultivation of diversified market players, control the electricity retail market power, and avoid the dominance of power retail companies by one category. It is encouraged that the power retail companies to expand service systems and enrich service models to provide users with more personalization, diversification and customization. They should create a comprehensive energy service system with electricity as the core, and continue to improve users' satisfaction.

\section{Conclusion}

In order to better promote the construction of the electricity retail market, it is necessary to accurately grasp the development situation and construction results of the electricity retail market, discover the current achievements and deficiencies in the construction, and continue to optimize and adjust the future market development path and direction. In view of the current situation of imperfect evaluation methods of electricity retail market construction, this paper proposes an effectiveness evaluation model, which can effectively evaluate the effectiveness of China's electricity retail market construction and guide the construction. The effectiveness of the proposed model is verified through case study. The main conclusions of this article are as follows.

The key factors for the effectiveness of the electricity retail market construction include the electricity retail market mechanism, electricity retail market openness, market concentration, market quality, market performance, electricity retail side service content diversity, and electricity retail market satisfaction, which can systematically measure the effectiveness of the electricity retail market construction. The evaluation index system launched from the seven major aspects can effectively measure the construction effectiveness of the designated electricity retail market and guide the development of the market.

Though a great deal of progress of Chinese electricity retail market is made, there are still many problems to be settled down. We should gradually strengthen the electricity retail market mechanism, improve the quality and performance of the market, bringing more convenience and satisfaction to electricity users.

\section{Acknowledgment}

The work described in this paper was supported by Science and technology project of State Grid Corporation of China "Research on Competitiveness Evaluation Technology and Analysis Model of Electricity Retail Market” (SGFJJY00GHJS1800020).

\section{References}

1. Peng X, Tao X. Cooperative game of electricity retailers in China's spot electricity market[J]. Energy, 2018, 145(FEB.15):152-170.

2. Han Z, Hanjie Y, Gengfeng L, et al. Quantitative Resilience Assessment under a Tri-Stage Framework for Power Systems[J]. Energies, 2018, 11(6): 1427.

3. Han Z, Gengfeng L, Hanjie Y, et al. Collaborative Optimization of Post-Disaster Damage Repair and Power System Operation[J]. Energies, 2018, 11(10).

4. Su W, Huang A. A game theoretic framework for a next-generation retail electricity market with high penetration of distributed residential electricity suppliers[J]. Applied Energy, 2014, 119(APR.15):341-350. 
5. Zhuang Y, Kang C, HU J. Quality of Electricity Retail Market and Its Comprehensive Evaluation. Power system automation[J]. 2009. 